\title{
Ubi pus, ibi evacua: Optimal Non-surgical Gallbladder Drainage Following Acute Cholecystitis
}

\author{
Amol Agarwal ${ }^{1,2} \cdot$ Paul J. Thuluvath ${ }^{1,2}$ \\ Published online: 13 July 2020 \\ (c) Springer Science+Business Media, LLC, part of Springer Nature 2020
}

Keywords Endoscopic gallbladder drainage $\cdot$ Acute cholecystitis

Acute cholecystitis (AC) is a common complication of gallbladder disease, with over 215,000 inpatient admissions for $\mathrm{AC}$ in the USA in 2012. Although the length of hospital stay for an episode of AC is decreasing, its incidence and associated healthcare costs have increased [1]. The gold standard treatment for AC, supported by recent international guidelines [2], is early laparoscopic cholecystectomy (LC). LC should occur prior to hospital discharge, when possible, because the risk of recurrent gallbladder-related events after an episode of AC in untreated patients reaches $~ 30 \%$ by 12 months [2]. When patients are unfit for surgery, an alternative treatment approach, gallbladder drainage (GBD), must be considered. Currently, placement of a percutaneous cholecystostomy (PC) tube, which is performed by an interventional radiologist, is recommended in non-surgical candidates [2]. Nevertheless, particularly for patients with cirrhosis who have ascites or coagulopathy, the percutaneous route may be hazardous due to oozing of ascitic fluid along the tract or poor wound healing after cholecystostomy tube removal, and rarely life-threatening bleeding from inadvertent puncturing of collateral blood vessels. Endoscopic methods of GBD have been developed, including endoscopic ultrasound-guided transmural gallbladder drainage (EUSGBD) and endoscopic retrograde cholangiography-guided endoscopic transpapillary gallbladder drainage (ETP-GBD) (Fig. 1). To date, the availability of either of these techniques is extremely variable, depending on local expertise, and the data supporting their use have been largely limited to small, retrospective studies.

Paul J. Thuluvath

thuluvath@gmail.com

1 Institute of Digestive Health and Liver Diseases, Mercy Medical Center, Baltimore, MD, USA

2 Department of Medicine, University of Maryland School of Medicine, Baltimore, MD, USA
In this issue of Digestive Diseases and Sciences, Sobani et al. [3] provide a well-written systematic review of the available literature on ETP-GBD. The two primary methods to perform ETP-GBD include the nasocholecystic option, in which a transpapillary nasobiliary tube traverses the cystic duct, or the cholecystoduodenal stenting procedure, an entirely internal procedure whereby a transpapillary (5-7 French) double pigtail stent is placed with one end in the gallbladder and the other end in the duodenum. The authors rightfully conclude that, despite a lack of comparative studies between the two transpapillary methods, the nasocholecystic option is poorly tolerated by patients and has fallen out of favor; it also carries the risk of nasopharyngitis if the nasobiliary tube is left in place for a prolonged period of time. Thus, for transpapillary drainage the authors clearly prefer the cholecystoduodenal stenting procedure, and most of their review is geared toward this method. Regardless of which transpapillary technique is used, the initial steps of biliary cannulation, sphincterotomy, bile duct clearance, and selective cystic duct cannulation are common for both endoscopic procedures. It is this latter step, cystic duct cannulation, which presents the greatest technical challenge and generally accounts for cases of technical failure. The procedure is particularly technically challenging when the cystic duct branches caudally from the common bile duct, creating an acute angle that the guidewire cannot easily traverse.

By all measures, ETP-GBD is a nascent technique with only 33 small studies including only a total of 1450 patients published. Since the global experience of percutaneous GBD in non-surgical patients is so much more well established, several important considerations would have to be addressed before moving ETP-GBD to mainstream clinical practice. First, does it achieve technical and clinical success in an acceptable proportion of cases, relative to the percutaneous route? Second, is it safe? 
Fig. 1 Graphical comparison of the two primary endoscopic methods for gallbladder drainage (Reprinted by permission from Elsevier: Oh, et al. GIE 2019;89:289-298)
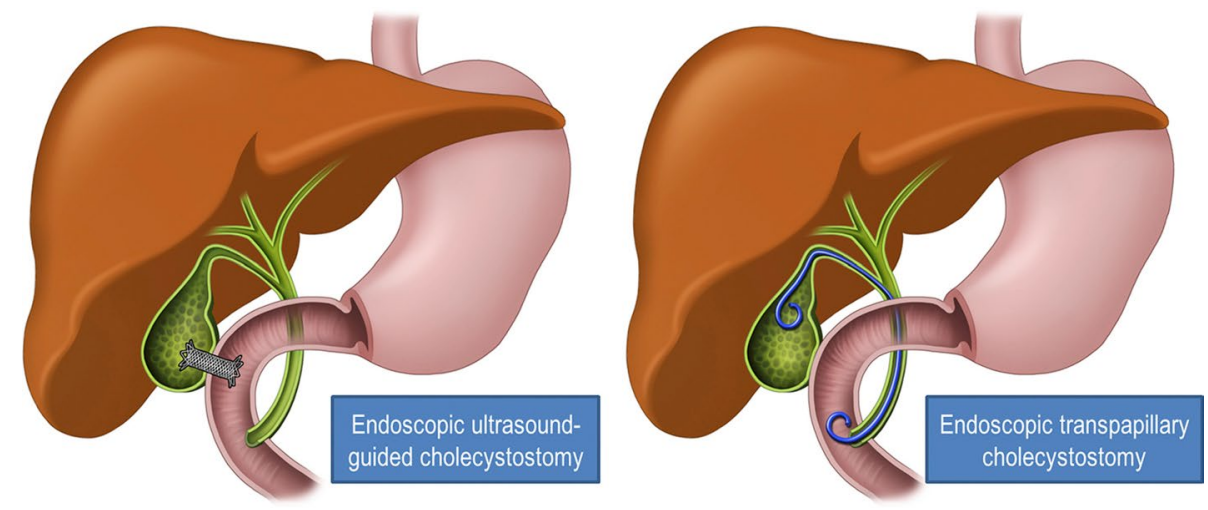

With regard to technical and clinical success, one of the meta-analyses cited in the review that evaluated 22 studies and 1223 patients demonstrated technical success rates of $83 \%$ and clinical success rates of $88.1 \%$ [4]. Although difficult to directly compare these results to those of percutaneous GBD due to selection bias to the analysis of largely retrospective data, the clinical success rates were numerically similar ( $89.3 \%$ for percutaneous GBD). Beyond initial clinical success, it would be useful to know how easily a subsequent LC is able to be performed. In a study of 192 patients undergoing either ETP-GBD or percutaneous GBD, there was a significantly lower rate of post-cholecystectomy complications in patients undergoing ETP-GBD [5]. Further, the ability to maintain gallbladder decompression with the ETP-GBD procedure demonstrated an impressive median 760 days of stent patency [6]. Another study, comparing 33 patients with transpapillary GBD compared with 29 patients with percutaneous GBD, showed that the rates of recurrent cholecystitis was $0 \%$ and $17.6 \%$, respectively [7]. These small studies suggest that transpapillary GBD can serve either as a bridge to $\mathrm{LC}$ if the patient becomes a surgical candidate, or as a palliative procedure for the remainder of the patient's life. In either situation, we suspect this would promote a far superior quality-of-life for the patient, as a percutaneous incision and external drainage bag are avoided.

The authors report that most of the adverse events are related to the performance of endoscopic retrograde cholangiography itself, which include pancreatitis and postsphincterotomy bleeding [3]. Nevertheless, there are also procedure-specific adverse events for ETP-GBD, specifically with regard to 1) attempted cannulation of the cystic duct, which can lead to cystic duct injuries and gallbladder perforation, and 2) placement of the cholecystoduodenal stent, which can migrate and clog, leading to cholangitis and/or liver abscess. Although most stents do get clogged, infectious complications are uncommon as biliary drainage generally occurs around the stents by a wicking action. Despite these risks, ETP-GBD compares favorably with PC, with significantly decreased odds of complications (13.3\% vs.
$39.5 \%$ ), mean time to clinical resolution (2.95 vs. 4.6 days), and rate of reintervention (13\% vs. 53\%) [8]. In a separate meta-analysis of safety in 3 studies, the pooled odds ratio for adverse events of endoscopic GBD compared to percutaneous drainage was 0.33 (95\% CI 0.14, 0.80) [9], although some of the cases in the endoscopic GBD were EUS-based procedures.

Although not the focus of their review, EUS-GBD is frequently mentioned because many of the studies on ETPGBD combine data on outcomes with those of EUS-GBD. This is unfortunate, as these endoscopic procedures are quite different. Nonetheless, since the data for EUS-GBD are favorable, this procedure has the potential to be superior to ETP-GBD. In a study comparing EUS-GBD to PC, both procedures showed comparable technical success $(97 \%$ in each group) and clinical success (100\% vs. 96\%), although EUS-GBD had lower post-procedure pain scores [10]. One of the few studies comparing EUS-GBD to ETP-GBD suggested superior clinical success with EUS-GBD (95\% vs. $76.3 \%$ ) [11]. This retrospective study, however, is not definitive due to selection bias.

In the present review, the authors propose a treatment algorithm for management of acute cholecystitis in non-surgical patients. They state that if the patient is not a potential surgical candidate in the future, then EUS-guided transmural gallbladder drainage should be performed. Although some preliminary data suggest technical and clinical superiority of EUS-GBD compared with ETP-GBD, the choice of procedure for a specific patient needs to be individualized. For instance, although ascites is less of an issue with EUSGBD than with percutaneous GBD, severe ascites could make EUS-guided placement of a stent more difficult and problematic. Furthermore, placement of a lumen-apposing metal stent (LAMS) through the duodenum or stomach would necessitate a repair of the defect, should an interval cholecystectomy be required; thus, transpapillary drainage has the benefit of "leaving the door open" for future surgical intervention should the patient's surgical candidacy improve. Finally, the monetary cost of EUS-GBD is greater. 
We propose starting with the intention of performing ETPGBD, but if technical issues are encountered during cystic duct cannulation, or in cases of duodenal obstruction, then in the same treatment session, the procedure can be converted to an EUS-guided LAMS placement. In terms of a patient's future surgical candidacy, there is still too much subjectivity, in our opinion, to use this as the main guiding principle in determining the type of endoscopic approach to perform. In our practice, we use the transmural route primarily as a salvage procedure for failed ETP-GBD, even for patients not likely to be future surgical candidates.

In summary, Sobani et al. provide a comprehensive scholarly overview of the available evidence supporting ETP-GBD as a safe and effective endoscopic technique for managing patients with $\mathrm{AC}$ who are not surgical candidates. If the cystic duct can be cannulated, the benefits of ETPGBD seem to be greater than either EUS-GBD or PC. For a definitive answer, however, we need a high-quality randomized controlled trial comparing these techniques, especially ETP-GBD to PC. Such a study may be difficult, but not impossible, to perform as it would require local expertise for transpapillary drainage, a sufficient number of AC patients who are not surgical candidates, and a standard definition of a non-surgical candidate agreed upon by surgeons, gastroenterologists, and interventional radiologists. With the rise in interventional endoscopy, the time is ripe to consider a multi-center randomized controlled trial to definitively answer these questions.

Funding None.

\section{Compliance with Ethical Standards}

Conflicts of interest The authors declared that they have no conflict of interest.

\section{References}

1. Wadhwa V, Jobanputra Y, Garg SK, Patwardhan S, Mehta D, Sanaka MR. Nationwide trends of hospital admissions for acute cholecystitis in the United States. Gastroenterol Rep. 2017;5:36-42.

2. Ansaloni L, Pisano M, Coccolini F, et al. WSES guidelines on acute calculous cholecystitis. World J Emerg Surg. 2016;11:25.

3. Sobani, Z, Ling C, Rustagi T. Endoscopic transpapillary gallbladder drainage for acute cholecystitis. Dig Dis Sci. (Epub ahead of print). https://doi.org/10.1007/s10620-020-06422-z.

4. Mohan BP, Khan SR, Trakroo S, et al. Endoscopic ultrasoundguided gallbladder drainage, transpapillary drainage, or percutaneous drainage in high risk acute cholecystitis patients: a systematic review and comparative meta-analysis. Endoscopy. 2020;52:96-106.

5. Kaura K, Bazerbachi F, Sawas T, Levy MJ, Martin JA, Storm AC, et al. Surgical outcomes of ERCP-guided transpapillary gallbladder drainage versus percutaneous cholecystostomy as bridging therapies for acute cholecystitis followed by interval cholecystectomy. HPB; 2019.

6. Lee TH, Park DH, Lee SS, et al. Outcomes of endoscopic transpapillary gallbladder stenting for symptomatic gallbladder diseases: a multicenter prospective follow-up study. Endoscopy. 2011;43:702-708.

7. Inoue T, Okumura F, Kachi K, et al. Long-term outcomes of endoscopic gallbladder stenting in high-risk surgical patients with calculous cholecystitis (with videos). Gastrointestinal Endosc. 2016;83:905-913.

8. Kedia P, Sharaiha RZ, Kumta NA, et al. Endoscopic gallbladder drainage compared with percutaneous drainage. Gastrointestinal Endosc. 2015;82:1031-1036.

9. Khan MA, Atiq O, Kubiliun N, et al. Efficacy and safety of endoscopic gallbladder drainage in acute cholecystitis: Is it better than percutaneous gallbladder drainage? Gastrointestinal Endosc. 2017;85:76-87.

10. Jang JW, Lee SS, Song TJ, et al. Endoscopic ultrasound-guided transmural and percutaneous transhepatic gallbladder drainage are comparable for acute cholecystitis. Gastroenterology. 2012;142:805-811.

11. Higa JT, Sahar N, Kozarek RA, et al. EUS-guided gallbladder drainage with a lumen-apposing metal stent versus endoscopic transpapillary gallbladder drainage for the treatment of acute cholecystitis (with videos). Gastrointestinal Endosc. 2019;90:483-492.

Publisher's Note Springer Nature remains neutral with regard to jurisdictional claims in published maps and institutional affiliations. 